Article

\title{
Accelerated Iterative Learning Control of Speed Ripple Suppression for a Seeker Servo Motor
}

\author{
Dongqi Ma *(D) and Hui Lin \\ School of Automation, Northwestern Polytechnical University, Xi'an 710029, China; linhui@nwpu.edu.cn \\ * Correspondence: madongqi0499@163.com; Tel.: +86-29-8843-1311
}

Received: 10 September 2018; Accepted: 2 October 2018; Published: 10 October 2018

\begin{abstract}
To suppress the speed ripple of a permanent magnet synchronous motor in a seeker servo system, we propose an accelerated iterative learning control with an adjustable learning interval. First, according to the error of current iterative learning for the system, we determine the next iterative learning interval and conduct real-time correction on the learning gain. For the learning interval, as the number of iterations increases, the actual interval that needs correction constantly shortens, accelerating the convergence speed. Second, we analyze the specific structure of the controller while applying reasonable assumptions pertaining to its condition. Using the $\lambda$-norm, we analyze and apply our mathematical knowledge to obtain a strict mathematical proof on the P-type iterative learning control and obtain the condition of convergence for the controller. Finally, we apply the proposed method for periodic ripple inhibition of the torque rotation speed of the permanent magnet synchronous motor and establish the system model; we use the periodic load torque to simulate the ripple torque of the synchronous motor. The simulation and experimental results indicate the effectiveness of the method.
\end{abstract}

Keywords: permanent magnet synchronous motor; speed ripple suppression; iterative learning control; learning interval adjustment; gain correction

\section{Introduction}

The permanent magnet synchronous motor has the advantages of high efficiency, high power density, and a high torque inertia ratio [1] and has been widely applied to the fields of aerospace, robotics, and transportation [2-5]. In recent years, as the requirements for system performance and accuracy have increased, how to suppress the speed ripple of the permanent magnet synchronous motor has become an active research area. In particular, in the direct drive system, because there is no transmission link involving gears, the rotation speed of the motor is relatively low, and the problems of low-frequency noise and vibration caused by the speed ripple are more severe. The torque ripple is the main source of the speed ripple. Based on extensive study, researchers worldwide have proposed multiple methods to suppress the torque ripple of the permanent magnet synchronous motor, which are mainly divided into two classes: Improving the main body design of the motor and optimizing the design of the software control algorithm.

The methods to improve the main body of the motor involve the stator slot [6], fractional slot [7], and toothless stator structure [8]. These methods can attenuate the speed ripple to some extent, but the design challenges are numerous, and the design cost is high. Regarding the aspect of software control, a previous study [9] adopted an adaptive filter to filter the feedback current and improve the performance of the current loop. Based on a PI controller, Zhang et al. [10] superimposed an oscillating controller and used the torque current, generated by this controller, to counteract the load torque ripple. Chu et al. [11] adopted a speed-loop control strategy using a PI regulator and a repetitive controller in parallel. Without affecting the dynamic performance, this configuration improved the steady-state 
characteristics of the system and suppressed the periodic speed ripple, but no strict mathematical proof was given to ensure convergence. Another study [12] proposed a method based on the optimization of the voltage vector that improved the model predictive control and adopted duty cycle control, thus reducing the torque ripple, but the parameter selection of the cost function was found to be relatively challenging.

Iterative learning control [13-15] is a type of model-less control with a simple structure that does not need specific model parameters. Over a limited interval, only through a sufficient number of repeated iterations can the behavior of the execution object satisfy the expected requirements. Iterative learning control has important significance for situations with nonlinearity, strong coupling, challenging modeling, and the requirement of high-accuracy trajectory control [16]. The existing studies on the theory of iterative learning control concentrate mainly on proofs of convergence for the learning algorithm, the learning velocity, studies of the structural form for the learning law, the robustness of, and analysis method, for the iterative learning process, the initial values, and assorted engineering applications [17-23]. There have been few studies on iterative learning control of the servo system for the permanent magnet synchronous motor. Li et al. [24] proposed to adopt the method of cascade iterative learning control to revise the reference input signal and improve the system accuracy by iteratively correcting the reference input. Another study [25] combined sliding mode control and iterative learning and proposed a robust iterative learning control strategy using a performance weighting function to improve the system robustness.

In this paper, to address the speed ripple of a permanent magnet synchronous motor, we propose an accelerated iterative learning control. The characteristics of the proposed method are as follows: For the learning interval of this control, as the number of iterations increases, the actual interval that needs correction constantly shortens, which accelerates the convergence speed. In this time interval, we conduct real-time correction of the learning gain. Outside the correction interval, the error is within the allowable range; there is no need for correction, and we directly apply the learned control input. That is, we constantly shorten the learning interval until learning is completed. This method reduces the overall computation amount by accelerating the learning speed. The proposed method not only accelerates the speed of iterative learning but also increases the tracking accuracy of the system. In this paper, we give a strict mathematical proof and verify the effectiveness and accuracy of the theory through simulation and experiment.

\section{Description and Analysis of the Question}

Consider a class of nonlinear systems:

$$
\left\{\begin{array}{l}
\dot{x}_{k}(t)=f\left(x_{k}(t), t\right)+B u_{k}(t) \\
y_{k}(t)=C x_{k}(t)+D u_{k}(t)
\end{array}\right.
$$

where $t \in\left[0, T_{L}\right] ; k$ is the number of iterations; $x_{k} \in R^{n}, u_{k}(t) \in R^{r}$, and $y_{k}(t) \in R^{m}$ are the state variables, control variables, and output variables of the system, respectively; and $B, C$, and $D$ are the matrices of the corresponding dimension.

Control objective: Design a controller for the system and let the system output track the expected trajectory under this controller.

For the convenience of proof, we make reasonable assumptions for the algorithm:

Assumption 1. The unknown nonlinear function $f(\cdot, \cdot)$ satisfies the Lipschitz condition, namely, there is a constant $L>0$ that satisfies

$$
\left\|f\left(x_{2}(t), t\right)-f\left(x_{1}(t), t\right)\right\| \leq L\left\|x_{2}(t)-x_{1}(t)\right\|
$$


Assumption 2. There is an ideal control $u_{d}(t)$ that makes the state and output of the system be the expected values of $x_{d}(t)$ and $y_{d}(t)$, respectively.

In the method proposed in this paper, we divide the interval $\left[0, T_{L}\right]$ into $\mathrm{N}$ equal sub-intervals, each with length $h$.

Let

$$
\begin{aligned}
& t_{0}=0, t_{N+1}=T_{L} \\
& t_{i+1}=t_{i}+h, i=0,1,2, \cdots, N
\end{aligned}
$$

We presume that the controlled object (1) adopts the P-type learning law:

$$
\begin{aligned}
& u_{k+1}(t)=u_{k}(t)+\Delta u_{k+1}(t) \\
& \Delta u_{k+1}(t)= \begin{cases}0, & 0 \leq t \leq t_{i_{k}} \\
K_{P} e_{k+1}(t) e^{-K_{E}\left(\frac{t_{i_{k}}-t}{T_{L}}\right),} & t_{i_{k}}<t \leq T_{L}\end{cases}
\end{aligned}
$$

where $K_{P}>0$ is the proportional learning gain, $K_{E}>0$ is the exponential correction factor, and $t_{i_{k}} \in\left[0, T_{L}\right]$ is the cut-off time (note: in the $(k+1)$ th iteration, the error in interval $\left[0, t_{i_{k}}\right]$ satisfies the allowable range, and the error in the residual interval $\left[t_{i_{k+1}}, T_{L}\right]$ does not satisfy the allowable range).

Let $\delta_{\left[t_{i_{k}}, T_{L}\right]}$ be the indicative function, namely,

$$
\delta_{\left[t_{i_{k}}, T_{L}\right]}(t)= \begin{cases}0, & t \notin\left[t_{i_{k}}, T_{L}\right] \\ 1, & t \in\left[t_{i_{k}}, T_{L}\right]\end{cases}
$$

According to Equation (4), Equation (5) can be rewritten as

$$
\begin{gathered}
u_{k+1}(t)=u_{k}(t)+K_{P} e^{-K_{E}\left(\frac{t_{i_{k}}-t}{T_{L}}\right)} \delta_{\left[t_{i_{k}}, T_{L}\right]} e_{k+1}(t) \\
e_{k+1}(t)= \begin{cases}0, & 0 \leq t \leq t_{i_{k}} \\
y_{d}(t)-y_{k+1}(t), & t_{i_{k}} \leq t \leq T_{L}\end{cases} \\
L_{i_{k}}=\left\{l \mid\left\|e_{k}\left[t_{i_{k}+l}, t_{i_{k}+l+1}\right]\right\|_{\lambda} \neq 0,0 \leq l \leq N-i_{k}\right\} \\
l_{i_{k}}= \begin{cases}\min L_{i_{k}}, & L_{i_{k}} \neq \phi \\
0, & L_{i_{k}}=\phi \\
t_{i_{k+1}}=t_{i_{k}+l_{i_{k}}}\end{cases}
\end{gathered}
$$

For the aforementioned accelerated P-type iterative learning control, which is constructed with gain correction, the learning control law is described as follows: We presume that the initial operation input is $u_{0}$, whose value is random in the entire operation interval $\left[0, T_{L}\right]$. In Equation (8), the introduction of $l_{i_{k}}$ is aimed at obtaining the boundary point $t_{i_{k}+l_{i_{k}}}$ that needs to be learned for the next iteration selection.

The steps of this method are as following:

Step 1: Calculate $e_{k+1}(t)$ using Equation (6);

Step 2: Calculate $L_{i_{k}}$ using Equation (7);

Step 3: Calculate $l_{i_{k}}$ using Equation (8);

Step 4: Calculate $t_{i_{k+1}}$ using Equation (9);

Step 5: Calculate $u_{k+1}(t)$ using Equation (5);

Step 6: Ending this iteration and starting the next. And repeat step 1-5. 
The structure of the control law (3) indicates that the interval that has been learned previously does not require continued learning, and we only need to learn the interval of the expected trajectory that is not completely tracked. As the number of iterations $k$ increases, the required length of the learning interval gradually approaches zero. The controller block diagram is showing as Figure 1.

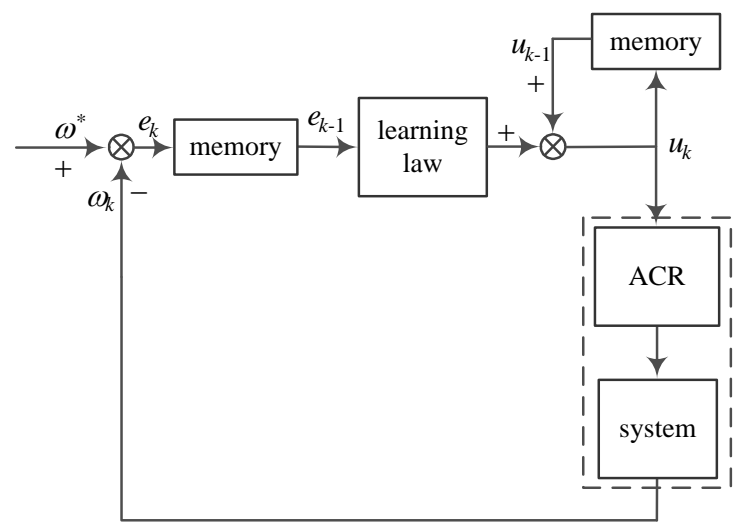

Figure 1. The block diagram of the controller.

Lemma 1. (Bellman-Gronwall inequality) [26]: Let $f_{1}(t), g_{1}(t)$, and $h_{1}(t)$ be nonnegative continuous functions in interval $\left[0, T_{L}\right]$. Moreover, there is a nonnegative constant $\eta$ that satisfies.

Thus, $f_{1}(t) \leq h_{1}(t)+\int_{0}^{t} \eta f_{1}(\tau) d \tau+\int_{0}^{t} g_{1}(t) d \tau$

$$
f_{1}(t) \leq h_{1}(t)+\int_{0}^{t} \exp (\eta \cdot(t-\tau))\left[\eta h_{1}(\tau)+g_{1}(\tau)\right] d \tau
$$

Theorem 1. If the system described by Equations (1) and (3) satisfies the conditions

(I) $\rho=\left\|\left(I+D K_{P} e^{K_{E}}\right)^{-1}\right\|<1$ and

(II) $x_{k}(0)=x_{d}(0),(k=0,1,2, \cdots)$

then

$$
\left\|\delta_{\left[t_{i_{k}}, T_{L}\right]} e_{k+1}(t)\right\|_{\lambda} \leq \rho\left\|\delta_{\left[t_{i_{k}}, T_{L}\right]} e_{k}(t)\right\|_{\lambda}
$$

when $k \rightarrow \infty$, the output of system $y_{k}(t)$ converges to the expected trajectory $y_{d}(t)$ in $\left[0, T_{L}\right]$, i.e.,

$$
\lim _{k \rightarrow \infty} y_{k}(t)=y_{d}(t)\left(t_{i} \in\left[0, T_{L}\right]\right)
$$

Proof. We presume that at the $(k+1)$ th iteration, the control time point stops at $t=t_{i_{k+1}}$; Equation (6) indicates that $y_{k+1}(t)=y_{k}(t)\left(t \in\left[0, t_{i_{k+1}}\right]\right)$. Therefore, when $t \in\left[t_{i_{k+1}}, T_{L}\right]$, the error can be expressed as

$$
\delta_{\left[t_{i_{k}}, T_{L}\right]} e_{k+1}(t)=y_{d}(t)-y_{k+1}(t)
$$

When $t \in\left[t_{i_{k+1}}, T_{L}\right]$, from Equation (1) and condition (II), we obtain Equation (12):

$$
\begin{aligned}
e_{k+1}(t) & =y_{d}(t)-y_{k+1}(t)=\left(y_{d}(t)-y_{k}(t)\right)-\left(y_{k+1}(t)-y_{k}(t)\right) \\
& =e_{k}(t)-C\left(x_{k+1}(t)-x_{k}(t)\right)-D\left(u_{k+1}(t)-u_{k}(t)\right) \\
& \left.=e_{k}(t)-C \int_{0}^{t} f\left(x_{k+1}(\tau), \tau\right)-f\left(x_{k}(\tau), \tau\right)\right) d \tau \\
& -C B \int_{0}^{t}\left(u_{k+1}(\tau)-u_{k}(\tau)\right) d \tau-D\left(u_{k+1}(t)-u_{k}(t)\right) \\
& =e_{k}(t)-C\left(\int_{0}^{t} f\left(x_{k+1}(\tau), \tau\right)-f\left(x_{k}(\tau), \tau\right)\right) d \tau \\
& -C B \int_{0}^{t} K_{P} e^{-K_{E}\left(\frac{t_{i_{k}}-\tau}{T_{L}}\right)} \delta_{\left[t_{i_{k}}, T_{L}\right]} e_{k+1}(\tau) d \tau-D K_{P} e^{-K_{E}\left(\frac{t_{i_{k}}-t}{T_{L}}\right)} \delta_{\left[t_{i_{k}}, T_{L}\right]} e_{k+1}(t)
\end{aligned}
$$


Rewritten Equation (12) as

$$
\begin{aligned}
& \delta_{\left[t_{i_{k}}, T_{L}\right]} e_{k+1}(t) \\
& =\left(I+D K_{P} e^{-K_{E}\left(\frac{{ }_{i_{k}}-t}{T_{L}}\right)}\right)^{-1} \delta_{\left[t_{i_{k}}, T_{L}\right]} e_{k}(t) \\
& -\left(I+D K_{P} e^{-K_{E}\left(\frac{t_{i_{k}}-t}{T_{L}}\right)}\right)^{-1} C B K_{P} \int_{0}^{t} e^{-K_{E}\left(\frac{t_{i_{k}}-\tau}{T_{L}}\right)} \delta_{\left[t_{i_{k}}, T_{L}\right]} e_{k+1}(\tau) d \tau \\
& -\left(I+D K_{P} e^{-K_{E}\left(\frac{{ }_{i_{k}}-t}{T_{L}}\right)}\right)^{-1} \int_{0}^{t} C\left(f\left(x_{k+1}(\tau), \tau\right)-f\left(x_{k}(\tau), \tau\right)\right) d \tau
\end{aligned}
$$

We take the norm on both sides of Equation (13) and multiply by $e^{-\lambda t}, \lambda>0$. Moreover, we take the maximum on $t \in\left[0, T_{L}\right]$ and combine it with the Lipschitz condition to obtain

$$
\begin{aligned}
\left\|\delta_{\left[t_{i_{k}}, T_{L}\right]} e_{k+1}(t)\right\|_{\lambda} \leq & \rho\left\|\delta_{\left[t_{i_{k}}, T_{L}\right]} e_{k}(t)\right\|_{\lambda}+\rho L c \frac{1-e^{-\lambda T_{L}}}{\lambda}\left\|x_{k+1}(t)-x_{k}(t)\right\|_{\lambda} \\
& +\rho b \frac{1-e^{-\lambda T_{L}}}{\lambda}\left\|\delta_{\left[t_{i_{k}}, T_{L}\right]} e_{k+1}(t)\right\|_{\lambda}
\end{aligned}
$$

Here,

$$
\rho=\left\|\left(I+D K_{P} e^{K_{E}}\right)^{-1}\right\|, c=\|C\|, b=\sup _{t \in\left[0, T_{L}\right]}\left\|C B K_{P}\right\| e^{-K_{E}\left(\frac{t_{i_{k}}-t}{T_{L}}\right)}
$$

From Equation (1), we obtain

$$
\begin{aligned}
& x_{k+1}(t)-x_{k}(t) \\
& \left.=\int_{0}^{t} f\left(x_{k+1}(\tau), \tau\right)-f\left(x_{k}(\tau), \tau\right)\right) d \tau+B \int_{0}^{t}\left(u_{k+1}(\tau)-u_{k}(\tau)\right) d \tau \\
& \left.=\int_{0}^{t} f\left(x_{k+1}(\tau), \tau\right)-f\left(x_{k}(\tau), \tau\right)\right) d \tau+B \int_{0}^{t} K_{P} e^{-K_{E}\left(\frac{t_{i_{k}}-\tau}{T_{L}}\right)} \delta_{\left[t_{i_{k}}, T_{L}\right]} e_{k+1}(\tau) d \tau
\end{aligned}
$$

We take the norm on both sides of Equation (15) and use the Lipschitz condition to obtain

$$
\left.\left\|x_{k+1}(t)-x_{k}(t)\right\| \leq \int_{0}^{t} L\left\|x_{k+1}(\tau)-x_{k}(\tau)\right\|\right) d \tau+B K_{P} e^{K_{E}} \int_{0}^{t}\left\|\delta_{\left[t_{i_{k}}, T_{L}\right]} e_{k+1}(\tau)\right\| d \tau
$$

From the Bellman-Gronwall inequality, we obtain

$$
\left\|x_{k+1}(t)-x_{k}(t)\right\| \leq B K_{P} e^{K_{E}} \int_{0}^{t} e^{L(t-\tau)}\left\|\delta_{\left[t_{i_{k}}, T_{L}\right]} e_{k+1}(\tau)\right\| d \tau
$$

We multiply both sides of the equation above by $e^{-\lambda t}$, where $\lambda>0$, and take the maximum on $t \in\left[0, T_{L}\right]$ to obtain

$$
\begin{aligned}
& \left\|x_{k+1}(t)-x_{k}(t)\right\|_{\lambda} \leq B K_{P} e^{K_{E}} \int_{0}^{t} e^{(L-\lambda)(t-\tau)} d \tau\left\|\delta_{\left[t_{i_{k}}, T_{L}\right]} e_{k+1}(t)\right\|_{\lambda} \\
& \leq a \frac{1-e^{(L-\lambda) T_{L}}}{\lambda-L}\left\|\delta_{\left[t_{i_{k}}, T_{L}\right]} e_{k+1}(t)\right\|_{\lambda}
\end{aligned}
$$

where $a=\left\|B K_{P} e^{K_{E}}\right\|$.

In interval $\left[t_{i_{k}}, T_{L}\right]$, we introduce Equation (18) into Equation (14) to obtain

$$
\left\|\delta_{\left[t_{i_{k}}, T_{L}\right]} e_{k+1}(t)\right\|_{\lambda} \leq \rho\left\|\delta_{\left[t_{i_{k}}, T_{L}\right]} e_{k}(t)\right\|_{\lambda}+\left(a \frac{1-e^{(L-\lambda) T_{L}}}{\lambda-L}+\rho b \frac{1-e^{\lambda T_{L}}}{\lambda}\right)\left\|\delta_{\left[t_{i_{k}}, T_{L}\right]} e_{k+1}(t)\right\|_{\lambda}
$$


We select a sufficiently large $\lambda$ and let

$$
\left\|\delta_{\left[t_{i_{k}}, T_{L}\right]} e_{k+1}(t)\right\|_{\lambda} \leq \rho\left\|\delta_{\left[t_{i_{k}}, T_{L}\right]} e_{k}(t)\right\|_{\lambda}
$$

After numbers of iterations, we obtain

$$
\left\|\delta_{\left[t_{i_{k}}, T_{L}\right]} e_{k+1}(t)\right\|_{\lambda} \leq \rho^{k-1}\left\|\delta_{\left[t_{i_{k}}, T_{L}\right]} e_{1}(t)\right\|_{\lambda}
$$

Therefore,

$$
\lim _{k \rightarrow \infty} \sup _{t \in\left[t_{i_{k}}, T_{L}\right]}\left\|\delta_{\left[t_{i_{k}}, T_{L}\right]} e_{k}(t)\right\|_{\lambda}=0
$$

This result indicates that control law (3) adopts the appropriate scheme to shorten the learning interval and ensure monotonic convergence under the $\lambda$-norm. When $\lambda$ is sufficiently large, as the number of iteration increases, we track the expected trajectory in a piecewise manner and conduct real-time correction of the learning gain. Moreover, the interval length which decreases and ultimately approaches zero. Finally, over the entire interval $\left[0, T_{L}\right]$, the system output tracks the expected trajectory.

\section{Simulation Results and Analysis}

The seeker servo system can be simplified as the following control system of a permanent magnet synchronous motor:

$$
\left\{\begin{array}{l}
\frac{d \theta(t)}{d t}=\omega(t) \\
\frac{d \omega(t)}{d t}=\frac{p}{J} T(t)-\frac{p}{J} T_{L}(\omega, t)-\frac{B}{J} \omega(t)-\frac{F}{J} \theta(t)
\end{array}\right.
$$

where $T_{e}$ is the electromagnetic torque, $T_{\mathrm{L}}$ is the load torque, $\theta$ is the angular position of the motor, $\omega$ is the angular speed of the motor, $p=2$ is the number of pairs, $B=0.0001 \mathrm{Nms}$ is the viscous friction coefficient, $J=0.00284 \mathrm{kgm}^{2}$ is the equivalent moment of inertia, and $F=0.00001 \mathrm{Nm}$ is the coefficient of elasticity. The output variable is $\omega(t)$, the control variable is $T(t)$, and the initial conditions are $\theta(0)=0$ and $\omega(0)=0$. A previous study [27] noted that the influence of the torque ripple above the 6 th order on the rotation speed can be neglected; thus, we focus our analysis on the 1 st, 2 nd, and 6th harmonic torque of the torque ripple. The load torque can be expressed as

$$
T_{L}(t, \omega)=T_{L_{-} 0}(t)+T_{L_{-} 1} \cos \left(\omega t+\varphi_{1}\right)+T_{L_{-} 2} \cos \left(2 \omega t+\varphi_{2}\right)+T_{L_{-} 6} \cos \left(6 \omega t+\varphi_{6}\right)
$$

The parameters of torque ripple are set as follows: $T_{L_{-} 0}=1$ is the average load torque, $T_{L_{-} 1}=0.5$ is the first harmonic content of the torque ripple, $T_{L_{-} 2}=0.1$ is the second harmonic content of the torque ripple, and $T_{L_{-6}}=0.05$ is the sixth harmonic content of the torque ripple. The unit is $\mathrm{Nm}$, and the phase of harmonic torque in the simulation is zero.

Figure 2 shows the process of tracking trajectory of the system output for the motor control system under the 1st-6th iterative learning steps. In particular, the dotted line is the expected speed, the dashed line is the tracking curve under the traditional P-type iterative learning control $\left(K_{P}=0.1\right)$, and the solid line is the tracking curve under described by Equation (3) $\left(K_{P}=0.1\right.$ and $\left.K_{\mathrm{E}}=3\right)$. Figure 2a indicates that after the first iteration, the two control methods both have a relatively large error. Figure $2 \mathrm{c}$ shows the control effect after the third iteration. The convergence speed of the proposed method is faster, and the tracking error satisfies the system requirements. Figure $2 \mathrm{f}$ shows that for the traditional P-type iterative learning control, after the 6th iteration, the control effect also essentially satisfies the requirement, but the error is larger than that for the proposed method. 


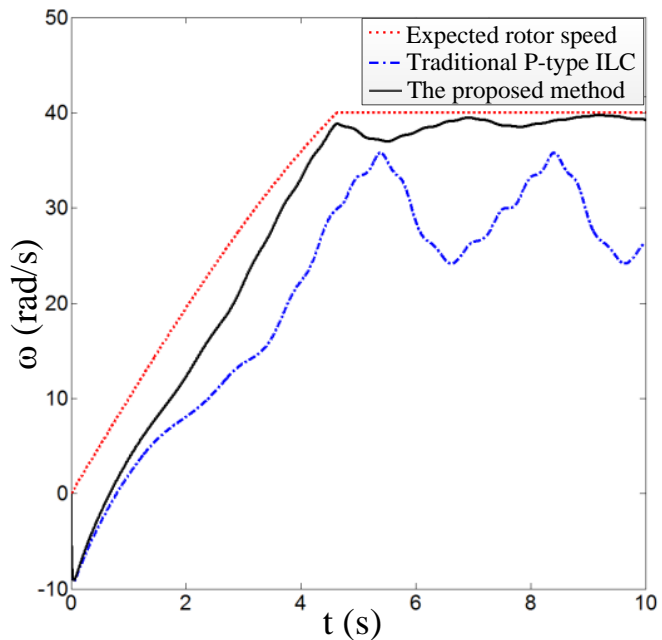

(a) First iteration

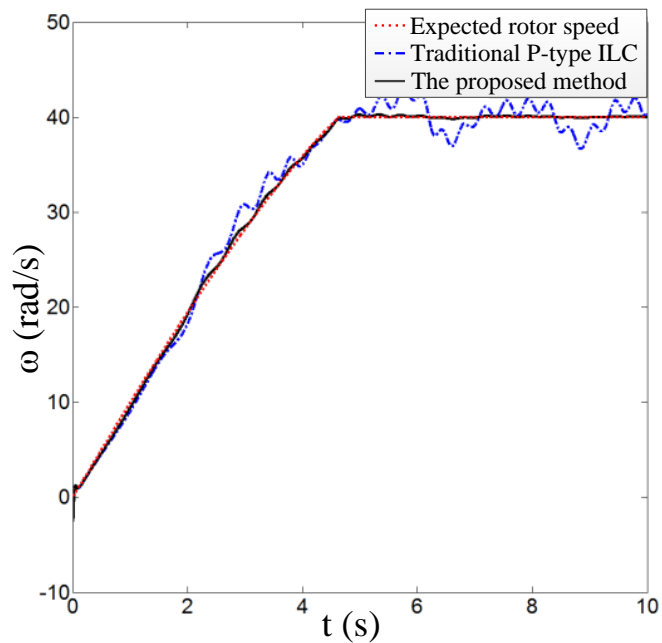

(c) Third iteration

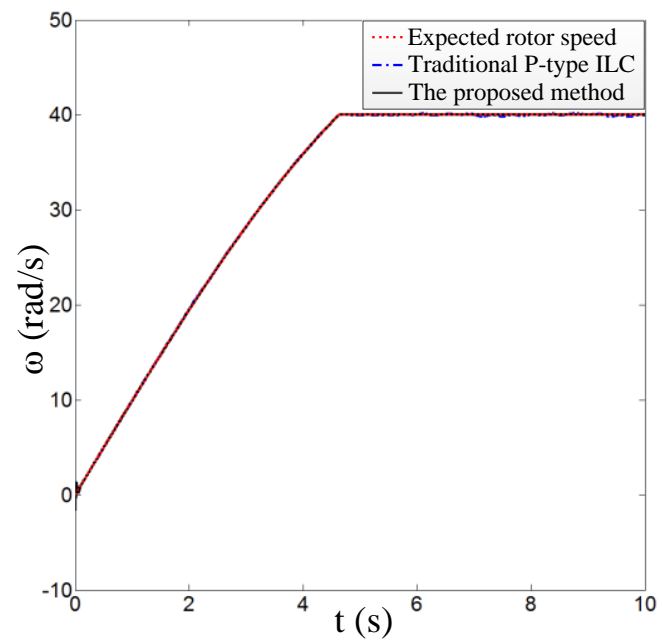

(e) Fifth iteration

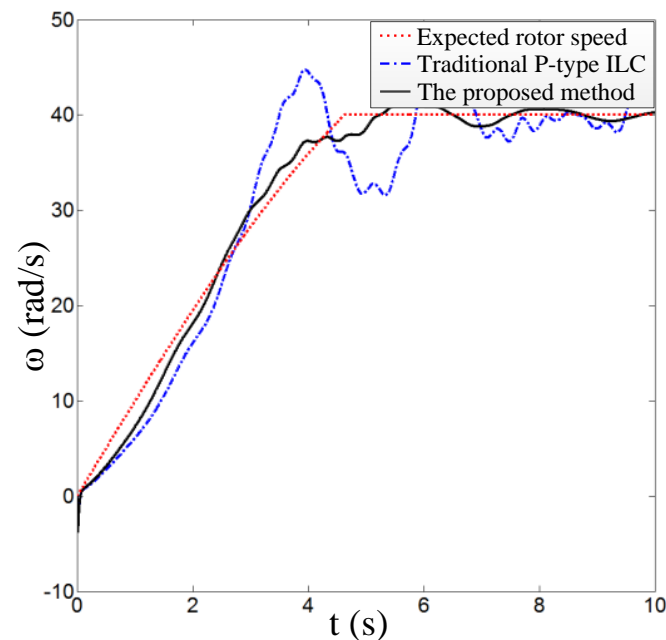

(b) Second iteration

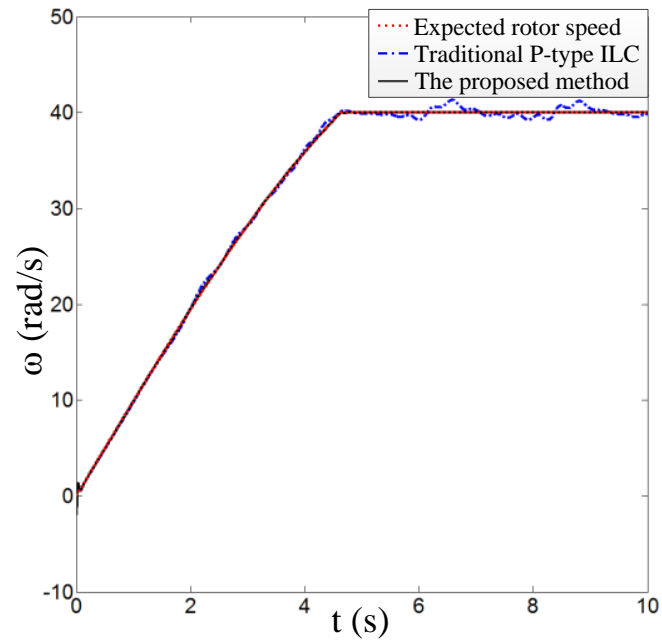

(d) Fourth iteration

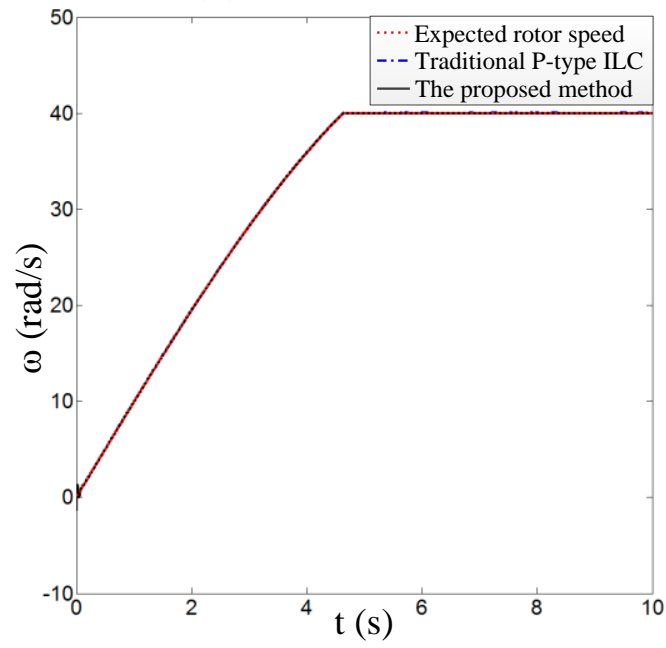

(f) Sixth iteration

Figure 2. Tracking curve of the motor angular speed in the simulation.

\section{Experimental Results and Analysis}

To verify the feasibility and effectiveness of the controller, we configure two experimental platforms in the laboratory (Figure 3). The proposed method is compared with the traditional PI control. 


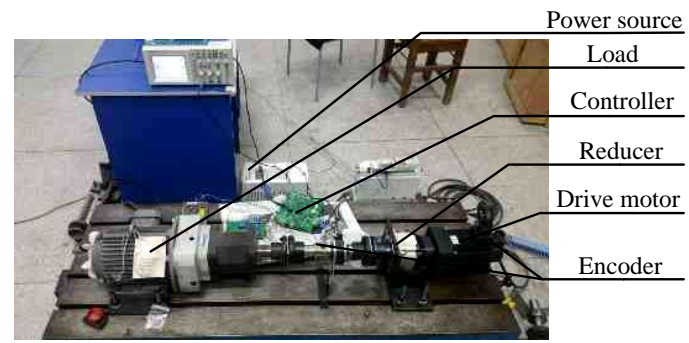

(a) Platform 1

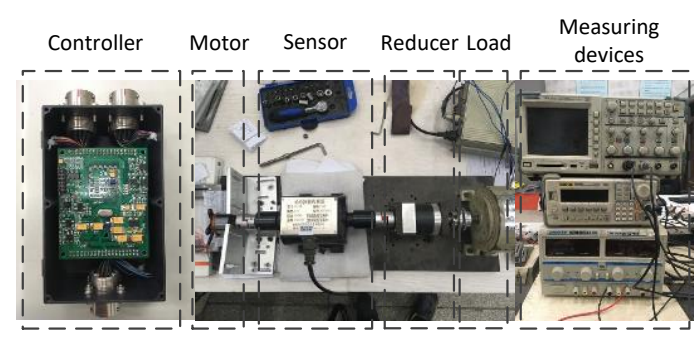

(b) Platform 2

Figure 3. Platform of the experimental system.

Figure 4 compares the proposed method and the traditional PI control and shows the effect of suppression under the two methods. In particular, Figure 4a shows the tracking situation of the motor angular speed. Relative to the speed closed-loop system, the periodic speed ripple exhibit relatively strong nonlinearity, and it is very challenging to completely respond only depending on the speed PI controller; moreover, the speed PI controller has some bandwidth limitations, which makes it susceptible to relatively high-order speed ripple, whereas the proposed method can satisfactorily suppress the ripple. The tracking error in Figure $4 \mathrm{~b}$ reinforces this point.

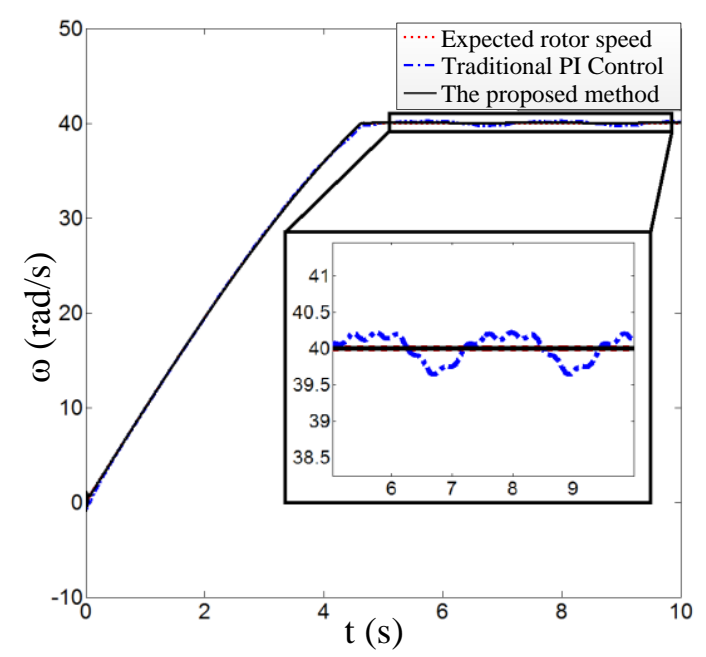

(a) Tracking curve of the two methods

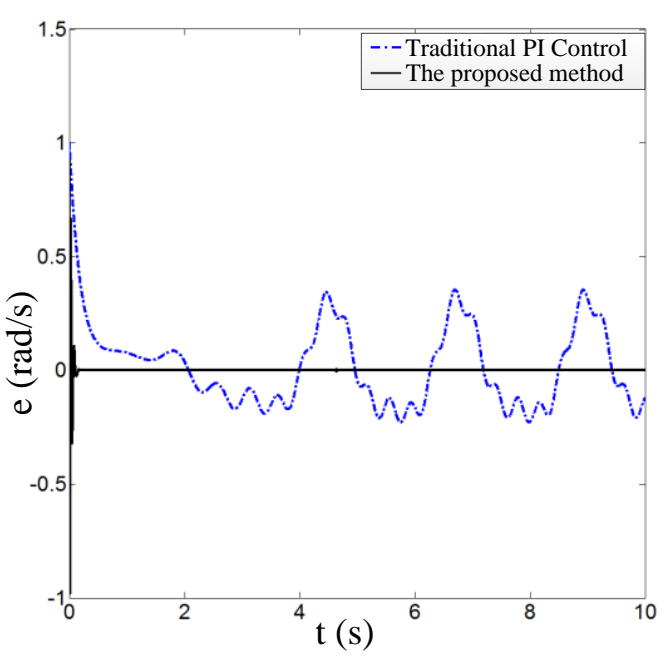

(b) Tracking error of the two methods

Figure 4. Tracking curve of the motor angular speed in the experiment.

\section{Conclusions}

In this paper, due to the requirements of a modern seeker servo system of fast velocity and high accuracy, we design an iterative learning control of gain correction with an adjustable learning interval. First, according to the system error of the current iteration, the next iterative learning interval is determined, and the learning gain is corrected in real time. With the increase of the number of iterations, the learning interval which needs to be corrected is shortened continuously. Then, the structure of the controller and its reasonable assumptions are analyzed. The closed loop P-type iterative learning control is strictly proved by applying the knowledge of mathematics, and the convergence condition of the controller is obtained in sense of $\lambda$-norm. Then, we use the P-type iterative learning law as an example and analyze the specific structure of the controller and its reasonable assumption conditions. Finally, we apply the proposed method to suppress the periodic ripple in the motor speed of the permanent magnet synchronous motor, and we establish the system model. We use the periodic load torque to simulate the ripple torque of the permanent magnet synchronous motor. The simulation and experimental results indicate the following: The proposed method not only accelerates the speed of iterative learning but also increases the tracking accuracy of the system. In upcoming studies, 
we plan to analyze the D-type and PD-type iterative learning control and incorporate other intelligent algorithms, such as sliding mode control, fuzzy control, and the neural network algorithm, with the accelerated iterative learning control. We can then use their respective advantages to control this complex system better.

Author Contributions: H.L. conceived the main idea of the proposed method. D.M. performed the experiments, analyzed the data and wrote the paper.

Funding: This work was funded by the National Natural Science Foundation of China (No. 51777170).

Acknowledgments: The authors would like to thank the reviewers for their constructive comments, which greatly helped to improve this paper.

Conflicts of Interest: The authors declare no conflict of interest.

\section{References}

1. Ryongho, J.; Zhanshan, W.; Chaomin, L.; Myongguk, J. Adaptive robust speed control based on recurrent elman neural network for sensorless PMSM servo drives. Neurocomputing 2017, 227, 131-141.

2. Slimane, M.; Demba, D.; Mohammed, M.; Claude, D.; Antoni, A. PMSM drive position estimation: Contribution to the high-frequency injection voltage selection issue. IEEE Trans. Energy Convers. 2015, 30, 349-358.

3. Vrkalovic, S.; Teban, T.A.; Borlea, I.D. Stable Takagi-Sugeno fuzzy control designed by optimization. Int. J. Artif. Intell. 2017, 15, 17-29.

4. Glowacz, A. Acoustic based fault diagnosis of three-phase induction motor. Appl. Acoust. 2018, 137, 82-89. [CrossRef]

5. Ramanathan, P.; Mangla, K.K.; Satpathy, S. The determination of combustion engine condition and reliability using oil analysis by MLP and RBF neural networks. Measurement 2017, 115, 557-572.

6. Fan, Z.N.; Han, L.; Liao, Y.; Xie, L.D.; Wen, K.; Wang, J.; Dong, X.C.; Yao, B. Effect of damper winding and stator slot skewing structure on no-load voltage waveform distortion and damper bar heat in large tubular hydro generator. IEEE Access 2018, 6, 22281-22291. [CrossRef]

7. Chung, S.U.; Kim, J.W.; Chun, Y.D.; Woo, B.C.; Hong, D.K. Fractional slot concentrated winding PMSM with consequent pole rotor for a low-speed direct drive: Reduction of rare earth permanent magnet. IEEE Trans. Energy Convers. 2015, 30, 103-109. [CrossRef]

8. Min, S.G.; Sarlioglu, B. Advantages and characteristic analysis of slotless rotary pm machines in comparison with conventional laminated design using statistical technique. IEEE Trans. Transp. Electrif. 2018, 4, 517-524. [CrossRef]

9. Wang, D.W.; Song, C.; Zhou, W. Adaptive filter applied in optimizing current loop of permanent magnet synchronous motor. Trans. China Electrotechn. Soc. 2014, S1, 90-94. (In Chinese)

10. Zhang, W.J.; Huang, S.D.; Gao, J.; Xiao, L. Suppression of speed ripples of permanent magnetic synchronous motor for compressor application. Control Theory Appl. 2013, 48, 27-29. (In Chinese)

11. Chu, J.B.; Hu, Y.W.; Huang, W.X.; Yang, J.F. Suppressing speed ripples of permanent magnetic synchronous motor based on a method. Trans. China Electrotechn. Soc. 2009, 24, 43-49. (In Chinese)

12. Yin, F.B.; Hua, W.; Huang, W.T.; Zhou, Z.Q. Voltage vector optimization-based model predicted torque control of flux-reversal permanent magnet machines. Proc. CSEE 2017, 37, 6524-6533. (In Chinese)

13. Arimoto, S.; Kawmura, S.; Miyazaki, F. Bettering operation of robotics by learning. J. Robot. Syst. 1984, 12, 123-140. [CrossRef]

14. Lan, T.Y.; Yan, F.; Lin, H. Iterative learning control with forgetting factor for urban road network. J. Control Sci. Eng. 2017, 2017, 1-7. [CrossRef]

15. Chi, R.H.; Hou, Z.S.; Xu, J.X. Adaptive ILC for a class of discrete-time systems with iteration-varying trajectory and random initial condition. Automatica 2008, 44, 2207-2213. [CrossRef]

16. Lin, H.; Wang, L. Iterative Learning Control Theory; $X_{i}$ an Northwestern Polytechnical University Press: Xi'an, China, 1998.

17. Deyuan, M.; Kevin, L.M. Convergence of iterative learning control for SISO non-repetitive systems subject to iteration-dependent uncertainties. Automatica 2017, 79, 167-177. 
18. Ardakani, M.; Khong, S.Z.; Bernhardsson, B. On the convergence of iterative learning control. Automatica 2017, 78, 266-273. [CrossRef]

19. Liu, S.D.; Wang, J.R. Fractional order iterative learning control with randomly varying trial lengths. J. Frankl. Inst. 2017, 354, 967-992. [CrossRef]

20. Altın, B.; Willems, J.; Oomen, T.; Barton, K. Iterative learning control of iteration-varying systems via robust update laws with experimental implementation. Control Eng. Pract. 2017, 62, 36-45. [CrossRef]

21. Tong, D.S.; Goele, P.; Jan, S. Multi-objective iterative learning control using convex optimization. Eur. J. Control 2017, 33, 35-42.

22. Chen, Y.Y.; Chu, B.; Christopher, T.F. Generalized norm optimal iterative learning control: Constraint handling. IFAC-PapersOnLine 2017, 50, 13396-13401. [CrossRef]

23. Preitl, S.; Precup, R.E.; Fodor, J.; Bede, B. Iterative feedback tuning in fuzzy control systems. Theory and Applications. Acta Polytech. Hung. 2006, 3, 81-96.

24. Li, B.Q.; Wu, C.; Lin, H. A high-precision position servo system of permanent magnet synchronous motors with reference input iterative learning. Proc. CSEE 2012, 32, 96-102.

25. Liu, J.; Li, H.W.; Deng, Y.T. Torque ripple minimization of PMSM based on robust iterative learning control. IEEE Trans. Power Electron. 2017, 25, 2645-2660.

26. Ruan, X.E.; Zhao, J.Y. Pulse compensated iterative learning control to nonlinear systems with initial state uncertainty. Control Theory Appl. 2012, 29, 993-1000.

27. Rupar, U.; Lahajnar, F.; Zajec, P. Iterative-learning-based torque-ripple compensation in a transverse flux motor. IET Control Theory Appl. 2012, 6, 341-348. [CrossRef]

(C) 2018 by the authors. Licensee MDPI, Basel, Switzerland. This article is an open access article distributed under the terms and conditions of the Creative Commons Attribution (CC BY) license (http:/ / creativecommons.org/licenses/by/4.0/). 